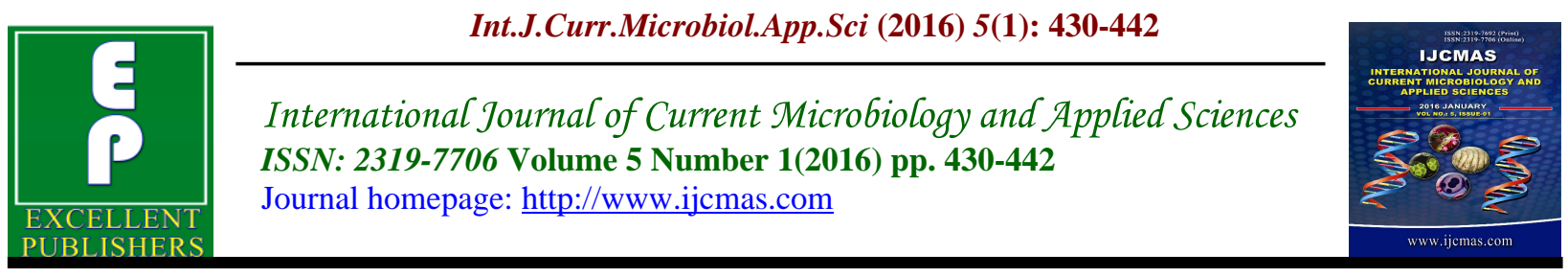

Original Research Article

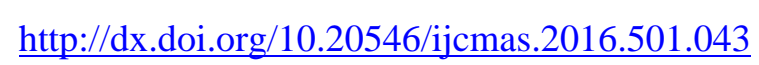

\title{
Loop-Mediated Isothermal Amplification (LAMP) Assay for Rapid Detection of Bacillus cereus Toxin Genes
}

\author{
Iychettira Machaiah Mandappa, Prasanna Joglekar, \\ Munna Singh Thakur andHaravey Krishnan Manonmani*
}

Food Protectants and Infestation Control Department, CSIR-Central Food Technological Research Institute, Mysore - 570 020, Karnataka, India

*Corresponding author

\begin{abstract}
A B S T R A C T
Keywords

Bacillus cereus

LAMP,

Isothermal

amplification

Food matrix

Detection,

Toxin genes

Article Info

Accepted:

18 December 2015

Available Online:

10 January 2016

In this communication we describe a Loop mediated isothermal amplification (LAMP) based method for quick and effective detection of specific Bacillus cereus enterotoxin genes. Primers for the enterotoxin genes (hemolysin-A ( $h b l A)$, Enterotoxin-T ( bceT), Enterotoxin-FM (entFM/cwpFM) and Cytotoxin-K $(C y t K)$ of Bacillus cereus were designed using Primer explorer 4. Hydroxynaphthol blue (HNB), SYBR Green I and Calcein were used to enable visual detection. An attempt was made to quantify the DNA using Varioskan Flash microplate reader. The primers showed negligible cross reactivity with other food borne pathogens studied. The lowest concentration of standard DNA detected corresponded to $10^{2}$, $10^{2}$ and $10^{3}$ copies of DNA respectively with Calcein, SYBR Green I and HNB dyes. The DNA isolated directly from the food matrix was used to validate the applicability of the developed LAMP assay. Negligible food matrix interference was observed in most cases. The method is quick, accurate and economical for onsite analysis. HNB was suitable for visual detection of B. cereus in some spiked food samples. Calcein proved to be a better dye for detection of spiked food samples when read in a Varioskan flash reader. This is one of the first reports on LAMP based detection of Bacillus cereus. The method is suitable for directly testing of food samples without any enrichment techniques and therefore could find its application in food safety analysis onsite. Such a detection system will ensure better food safety and can also be extended to clinical diagnosis of pathogenic organisms.
\end{abstract}

\section{Introduction}

Bacillus cereus is a facultative anaerobic, spore-forming, motile microorganism that can be commonly isolated from food. It is known to produce four toxins, one emetic toxin and three potential diarrheal toxins (Ehling-schulz, et al., 2004; Samapundo, et al., 2011). The emetic syndrome is mainly characterized by vomiting $0.5-6 \mathrm{~h}$ after ingestion of the contaminated food. In the diarrheal syndrome, symptoms appear 8$16 \mathrm{~h}$ after ingestion and include abdominal pain and diarrhea. In general, both types of 
food-borne illness are relatively mild and usually do last not more than $24 \mathrm{~h}$ (Dierick et al., 2005). However, it is difficult to accurately determine the incidence of Bacillus cereus infection as most of the cases go unreported or misdiagnosed (Granum 2005). Bacillus cereus spores usually survive high temperature cooking and later multiply to cause the disease symptoms (Rajkovic et al., 2013). Detection of these toxin producing bacteria in food can check widespread contamination.

Presently PCR based methods (Gracias and McKillip 2011) and existing kits used for detection of $B$. cereus have been shown toyield equivocal results (Burgess and Horwood 2006). PCR based nucleic acid detection though sensitive; requires highly pure form of DNA which is difficult to obtain from food samples. Also, Taq polymerase used in PCR reactions is often inhibited by the presence of food isolates(Schrader et al., 2012).

Loop mediated isothermal amplification, first reported by Natomi et al., 2000 (Notomi et al., 2000a), is an alternative to the present PCR based nucleic acid detections. LAMP uses Bst polymerase, having strand displacement activity and therefore amplifies at a single temperature. Four to six primers, which identify different regions of the target gene, make the assay specific. LAMP based detection of pathogens is a good alternative and can complement PCR because of its simplicity, speed and accuracy(Parida et al., 2006). The primary characteristics of the LAMP assay are its ability to amplify nucleic acid under isothermal conditions at $65^{\circ} \mathrm{C}$ with Bst DNA polymerase; as a result it allows the use of simple and cost effective reaction equipment. The second characteristic is that LAMP has high specificity and high amplification efficiency, with DNA being amplified $10^{9}-10^{10}$ times in $15-60 \mathrm{~min}$. The high amplification efficiency of LAMP is attributed to no time loss of thermal change (as in PCR) because of its isothermal nature(Wang 2013).

In this communication we describe a LAMP assay to detect four toxin genes ( $h b l A, b c e T$, entFM / cwpFM and cyt $K$ ) of Bacillus cereus. This is one of the first reports on detection of $B$. cereus diarrheal toxins using LAMP method. The applicability of the developed method was tested with spiked food samples.

\section{Materials and Methods}

\section{Test Cultures and Media}

Bacillus cereus MTCC 1272 (reference culture) maintained on brain Heart infusion (BHI) agar supplemented with $0.1 \%$ glucose (HiMedia, Mumbai, India) and other test cultures (Table 1) used in the cross reactivity studies were procured from Institute of Microbial Technology (IMTECH), Chandigarh, India.

\section{Isolation of Bacillus Cereus Strains}

A total of 25 different types of food matrices including meat and meat products, milk and milk based products, cakes and pastries, fried snacks, egg and egg products, fast foods, raw vegetables, fermented foods, etc., were used in this study (Table 2). The choice of food sample for isolation and spiking studies was done in accordance to the FDA methods validation guidelines for microbial pathogens(Service 2011).

A homogeneous sample was prepared in $0.85 \%$ sterile saline. The isolation of the cultures was done as described by (Samapundo et al., 2011). Aliquots of 0.1 $\mathrm{mL}$ from appropriate dilutions were surface plated on B.cereus specific Polymyxin B sulphate pyruvate egg yolk mannitol 
bromothymol blue agar (HiMedia Laboratories, Mumbai, India) plates. The inoculated plates were incubated at $37^{\circ} \mathrm{C}$ for 24 - 48 h. Presumptive colonies of B. cereus were selected based on characteristic colony features (peacock blue colored colonies with a surrounding zone of egg yolk precipitation). Further they were tested for $\beta$-hemolytic activity on blood agar plates (HiMedia Laboratories, Mumbai, India) containing 5\% defribinated sheep blood. The isolated colonies were maintained on Brain Heart Infusion (HiMedia Laboratories, Mumbai, India) agar containing $0.1 \%$ glucose. The DNA isolated from these isolates was used as the template for LAMP reactions.

\section{Isolation of DNA}

DNA from bacterial cultures was isolated by phenol: chloroform method as described by (Sambrook et al., 1989). The DNA was checked for its purity and was quantified by measuring the absorbance ratio at 260/280 $\mathrm{nm}$ using a Biospectrometer-Basic, Eppendorf India Pvt Ltd.

\section{LAMP Primers}

A total of six sets of primers for each toxin producing genes of target pathogenic $B$. cereus genome were used in this study. They were designed using Primer Explorerversion 4 (http://primerexplorer.jp/ elamp4.0.0/index.htmL) software. Four sets of LAMP primers designed for the $h b l A$, cytK, bceT and entFM/cwpFM gene are given in Table 3. All oligonucleotide primers used in this study were synthesized by Sigma-Aldrich chemical Company, Bangalore, India.

\section{LAMP Assay}

LAMP assay was carried out as described by (Tomita et al., 2008a) with slight modifications, wherein a $25 \mu \mathrm{L}$ reaction mixture containing, $8 \mathrm{U}$ of Bst polymerase large fragment (New England Bio Labs, Beverly, MA, USA), $5 \mu \mathrm{L}$ of template DNA $\left(10^{1}-10^{8}\right.$ copies, preheated at $95^{\circ} \mathrm{C}$ for 5 min, chilled on ice) along with $1.6 \mu \mathrm{M}$ each of inner primer (FIP and BIP), $0.2 \mu \mathrm{M}$ each of outer primer (F3 and B3 primer). $0.8 \mu \mathrm{M}$ of each loop primer (LF and LB), $1.4 \mathrm{mM}$ of deoxynucleotide triphosphate, $0.5 \mathrm{M}$ of Betaine (Sigma, St Louis, MO, USA), 20 $\mathrm{mM}$ of Tris- $\mathrm{HCl}(\mathrm{pH} 8.8), 10 \mathrm{mM}$ of $\mathrm{KCl}$, $10 \mathrm{mM}$ of $\left(\mathrm{NH}_{4}\right)_{2} \mathrm{SO}_{4}, 8 \mathrm{mM} \mathrm{MgSO} 4$ and $0.2 \%$ Tween-20. The reaction mixture was incubated at $65^{\circ} \mathrm{C}$ for $60 \mathrm{~min}$ in a water bath and then heated at $80{ }^{\circ} \mathrm{C}$ for $2 \mathrm{~min}$ to terminate the reaction. All the reactions were performed in triplicates.

LAMP products were subjected to electrophoresis on a $1.5 \%$ agarose gel, visualized under UV light after ethidium bromide staining and documented in a gel documentation system (Cleaver scientific Ltd, microDoc unit, UK).

\section{Specificity of the LAMP Primers}

Specificity of LAMP primers designed was checked with genomic DNA isolated from other food- borne pathogens given in Table 1. The LAMP assay conditions used were the same as mentioned earlier.

\section{Sensitivity of LAMP Reaction}

Total DNA was isolated from overnight reference B.cereus culture. The DNA obtained was diluted in the concentration range of $10^{8}$ to $10^{1}$ copies and used to study the sensitivity of LAMP assay as given above. The copy number was calculated using the online portal htttp://cels.uri.edu/gsc/cndna.htmĹ.

\section{Analysis of LAMP Products}

Performance of three dyes HNB, Calcein 
and SYBR Green I used in the study were compared. The Quantitative estimation was carried out by measuring the colour change of Hydroxynaphthol blue (HNB) (SRL, Mumbai, India) at $120 \mu \mathrm{M}$ concentration, 1 $\mu \mathrm{L}$ SYBR Green I (Sigma Aldrich, Bangalore, India) at $1 \mathrm{X}$ concentration and Calcein (SRL, Mumbai, India) at $2 \mu \mathrm{M}$ concentration were used in analysis of the LAMP products. The readings were recorded at $15 \mathrm{~min}$ intervals, using a Varioskan Flash microplate reader (Thermo Fisher, Finland).

\section{Spiking of Food Samples to Study Matrix Interference}

A total of 25 different types of food samples mentioned in Table 2, spiked with $10^{2}$ to $10^{8}$ cells $\mathrm{g}^{-1}$ or $\mathrm{mL}$ food were used to study matrix interference. Aliquots of $1 \mathrm{~g}$ or $1 \mathrm{~mL}$ food sample were used for the study.

\section{Preparation of Spiked Food Samples for LAMP Testing}

The food matrices spiked with $10^{2}$ to $10^{8}$ cells $\mathrm{g}^{-1}$ or $\mathrm{mL}$ (aliquots of $1 \mathrm{~g}$ or $1 \mathrm{~mL}$ ) food after equilibration for $1 \mathrm{~h}$ were suspended in isotonic saline and centrifuged at $2000 \mathrm{~g}$ for $10 \mathrm{~min}$ to remove the food matrix. The supernatant was boiled for 15 min with lysis solution consisting of $20 \mathrm{mM}$ Tris- $\mathrm{HCl}$ (pH 8), 2 mM EDTA ( $\mathrm{pH} \mathrm{8)}$ and $1.2 \%$ Triton X-100 (Sowmya et al., 2012). The boiled sample was centrifuged for 10 $\min$ at $3000 \mathrm{~g}$ and supernatant was used directly for detection by LAMP.

\section{Results and Discussion}

Primer Design for $\boldsymbol{B}$. cereus Toxin Genes and LAMP Assay

LAMP primers generated from Primer explorer-V4, for the toxin genes ( $h b l A$, bceT, cytK, entFM/cwpFM) of B. cereus were checked for the 5' and 3' stability and the best primer set was chosen for our study (Table 3).

The LAMP assay was performed according to (Tomita et al., 2008a). The time dependence of the reaction for the $h b l A$ gene $\left(10^{4}\right.$ copies of DNA) at 15 min intervals was checked on an agarose gel $(1.5 \%)$. A distinct ladder like pattern was observed (Fig. 1).

\section{Evaluation of B.cereus Food Isolates}

DNA extracted from all the 25 food sample isolates was subjected to LAMP analysis to check for the presence of B.cereus diarrheal toxin genes. All the food sample isolates mentioned in Table 3 showed positive results for $h b l$ A toxin gene. 16 isolates of the 25 isolates studied showed postitive amplification for the $b c e T$ gene and entFM/cwpFM. 14 of the 25 isolates showed positive results for $c y t K$ gene (Table 2).

\section{Sensitivity and Competence of the Dyes}

Hydroxynapthol Blue, Calcein and SYBR Green I were compared in detecting reference B.cereus (MTCC 1272)diarrheal toxin genes.

Hydroxynapthol Blue: The colour change of HNB was in agreement with the pattern obtained in agarose gel (Fig. 1). The colour change from violet to blue (measured by absorbance at $650 \mathrm{~nm}$ ) was less at $15 \mathrm{~min}$ interval and was maximum at the end of $1 \mathrm{~h}$ which corresponded with the readings obtained in a Varioskan Flash microplate reader. DNA concentrations as low as $10^{3}$ copies of DNA could be read with HNB dye (Fig. 2). The colour change could be visually differentiated at the DNA concentrations studied (Fig 2E). 
Table.1 List of Test Cultures

\begin{tabular}{ll}
\hline Bacterial Species & MTCC $^{1}$ No \\
\hline Bacillus cereus & 1272 \\
Vibrio cholerae & 3906 \\
Salmonella parathypi & 735 \\
Listeria monocytogenes & 1143 \\
Streptococcus pneumoniae & 655 \\
Staphylococcus aureus & 96 \\
Yersinia enterocolitica & 859 \\
Escherichia coli & 729 \\
\hline
\end{tabular}

Microbial type cell culture collection, IMTECH, Chandigarh

Table.2 Analysis of Food Isolates for the Presence of B. Cereus Toxin

\begin{tabular}{lllllll}
\hline S.No & Food samples & Isolate & hblA & bceT & entFM & cytK \\
\hline 1 & Condensed Milk & Hkmber & & & & \\
2 & Milk & HkmBC2 & + & + & + & -3 \\
3 & Butter & HkmBC3 & + & - & - & + \\
4 & Curds & HkmBC4 & + & + & + & + \\
5 & Paneer & HkmBC5 & + & + & - & - \\
6 & Cheese & HkmBC6 & + & + & - & + \\
7 & Milk burfee & HkmBC7 & + & + & + & + \\
8 & Colored Rice & HkmBC8 & + & + & + & + \\
9 & White Rice & HkmBC9 & + & + & + & + \\
10 & Apple pie & HkmBC10 & + & - & + & + \\
11 & Chapatti & HkkmBC11 & + & - & + & + \\
12 & Noodles & HkmBC12 & + & + & + & - \\
13 & Chicken sausage & HkmBC13 & + & - & + & + \\
14 & Meat (Pork) & HkmBC14 & + & + & - & + \\
15 & Carrot & HkmBC15 & + & + & + & - \\
16 & Boiled potato & HkmBC16 & + & + & + & + \\
17 & Beetroot & HkmBC17 & + & + & + & - \\
18 & Tomato & HkmBC18 & + & - & - & - \\
19 & Orange juice & HkmBC19 & + & - & - & - \\
20 & Minced Chicken & HkmBC20 & + & + & + & + \\
21 & Samosa & HkmBC21 & + & + & + & + \\
22 & Panipuri & HkmBC22 & + & - & - & - \\
23 & Bhel puri & HkmBC23 & + & + & - & + \\
24 & White sauce & HkmBC24 & + & - & + & - \\
25 & Tomato ketchup & HkmBC25 & + & - & - & - \\
\hline
\end{tabular}

\footnotetext{
${ }^{1}$ Microbial type cell culture collection, IMTECH, Chandigarh

${ }^{2}$ Positive amplification +

${ }^{3}$ Negative amplification -
} 
Table.3 List of LAMP Primers

\begin{tabular}{cll}
\hline Genes & Primer & Sequence \\
\hline & hbl F3 & TGCTATTTTGGGTCTACCAAT \\
hbl B3 & GGACATATAAGTAAGAGCGTTAA \\
$(\text { L20441.1 })^{4}$ & hbl FIP & ACGTAATTCTGCTAATAAAGGCTCTTTGGCGGTATTATAGTGGGA \\
& hbl BIP & AACCTTAAATCGTGTAGTTGGAGTTATCATCAAGCGCCTTGTC \\
& hbl LF & TGCTATTTTGGGTCTACCAAT \\
& cytkF3 & CTAGCGTATCTTATCAACTTGG \\
cytK gene & cytkB3 & CCGTTAAAGAATACGTTCCAT \\
$($ DQ019311.1) & cytkFIP & GACCAGTTGCACCAGCTTCATGGCTCTGTTAAAGCTTCTG \\
& cytkBIP & AAGTCACTTGGTCTGACTCTGTTTACGTTTTTGTCGTTTGG \\
& cytk LB & CGCTAGGGCCATTAGGCGT \\
& bceT F3 & GAAGTAATAAGCGTACCATCTG \\
& bceT B3 & GAAGTAATAAGCGTACCATCTG \\
bceT gene & bceT FIP & ACGGTCTAGCTAACGCTTCAATTTAACTGGATGCTGTTATTGACT \\
(D17312.1) & bceT BIP & GGTTACCACCACTTCCAGAAAG-TTCTTTTCTTTCGCCCATG \\
& bceT LB & GAAGTAATAAGCGTACCATCTG \\
& & \\
& entfmF3 & GGTTATGTAAGTGCAGACTTC \\
entfmB3 & TCAAAACCAGCAGGTGTT \\
entFM gene & entfmFIP & CGTCTTTACCTGGTTGTTAACGGTTAAGTTTGTAAAAGGCGGA \\
(AY789084.1) & entfmBIP & CCAACAACAGGTGGAGATACATCGCAGTTCTGTATGGTGAAC \\
& entfmLF & TCGCTGGATTCGCTAGATCTTT \\
& entfmLB & GGTTATGTAAGTGCAGACTTC \\
\hline
\end{tabular}

Figure 1. Agarose Gel Showing Positive LAMP Amplification for HBLA Gene at Different Intervals of Time

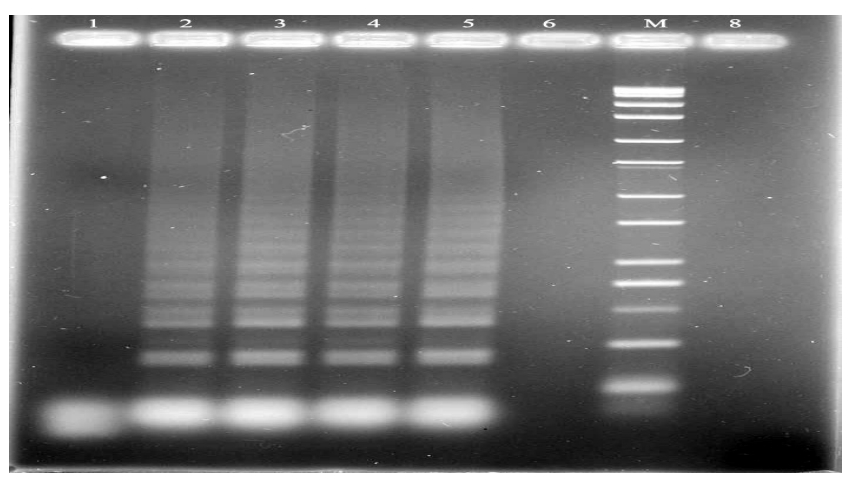

Lane 1: 0 min; Lane 2: $15 \mathrm{~min}$; Lane 3: $30 \mathrm{~min}$; Lane 4: $45 \mathrm{~min}$; Lane 5: $60 \mathrm{~min}$; Lane 6: Control and Lane M: $100 \mathrm{~kb}$ Marker

${ }^{4}$ GenBank Acession Number 
Figure.2 LAMP Analysis of Toxin Genes of B. Cereus with HNB
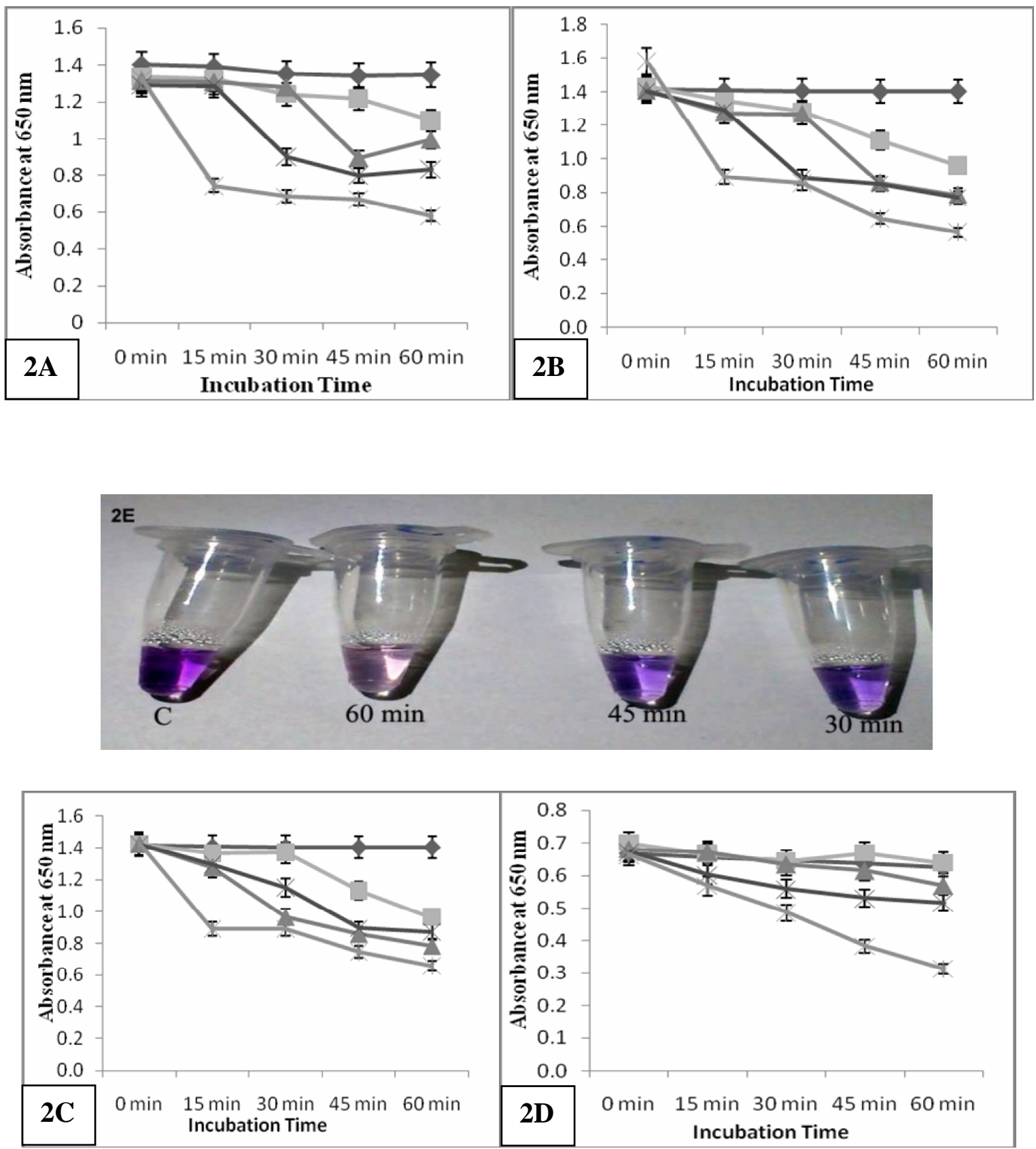

LAMP analysis of 2A) $h b l A$ gene; 2B) bceT gene; 2C) entFM/cwpFM and 2D) $c y t K$ genes at 15 min intervals with varying concentrations of standard Bacillus cereus DNA.

2E) color change in tubes containing $10^{4}$ copies of DNA under UV light at varying incubation times:Tube Ccontrol, Tube 1: $60 \mathrm{~min}$, Tube 2: $45 \mathrm{~min}$, Tube 3: $30 \mathrm{~min}$.

$(\diamond)$ Control, ( $\mathbf{\square}) 10^{1}$ copies, ( $\left.\mathbf{\Delta}\right) 10^{4}$ copies, (x) $10^{7}$ copies, $(*) 10^{8}$ copies 
Figure.3 LAMP Analysis of Toxin Genes of B. Cereus with Calcein
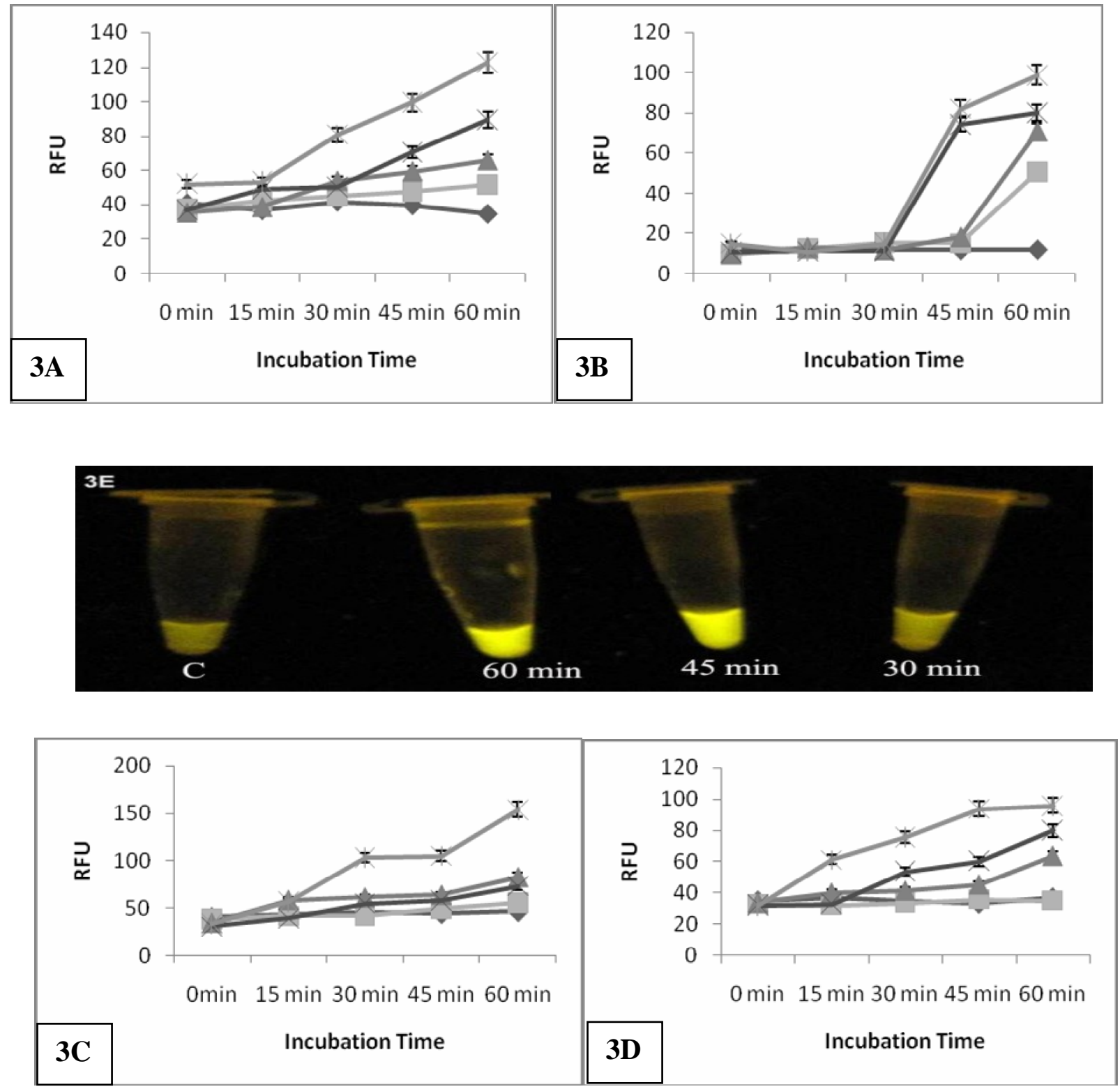

LAMP analysis of 3A) hblA gene; 3B) bceT gene; 3C) entFM/ cwpFM and 3D) cytK genes at 15 min intervals with varying concentrations of standardBacillus cereus DNA.

$3 \mathrm{E})$ color change in tubes containing $10^{4}$ copies of DNA under UV light at varying incubation times: Tube C: control, Tube 1: $60 \mathrm{~min}$, Tube 2: $45 \mathrm{~min}$, Tube 3: $30 \mathrm{~min}$.

$(\diamond)$ Control, ( $\mathbf{\square}) 10^{1}$ copies, $(\mathbf{\Delta}) 10^{4}$ copies, (x) $10^{7}$ copies, $(*) 10^{8}$ copies 
Figure.4 LAMP Analysis of Toxin Genes of B. Cereus with SYBR Green I
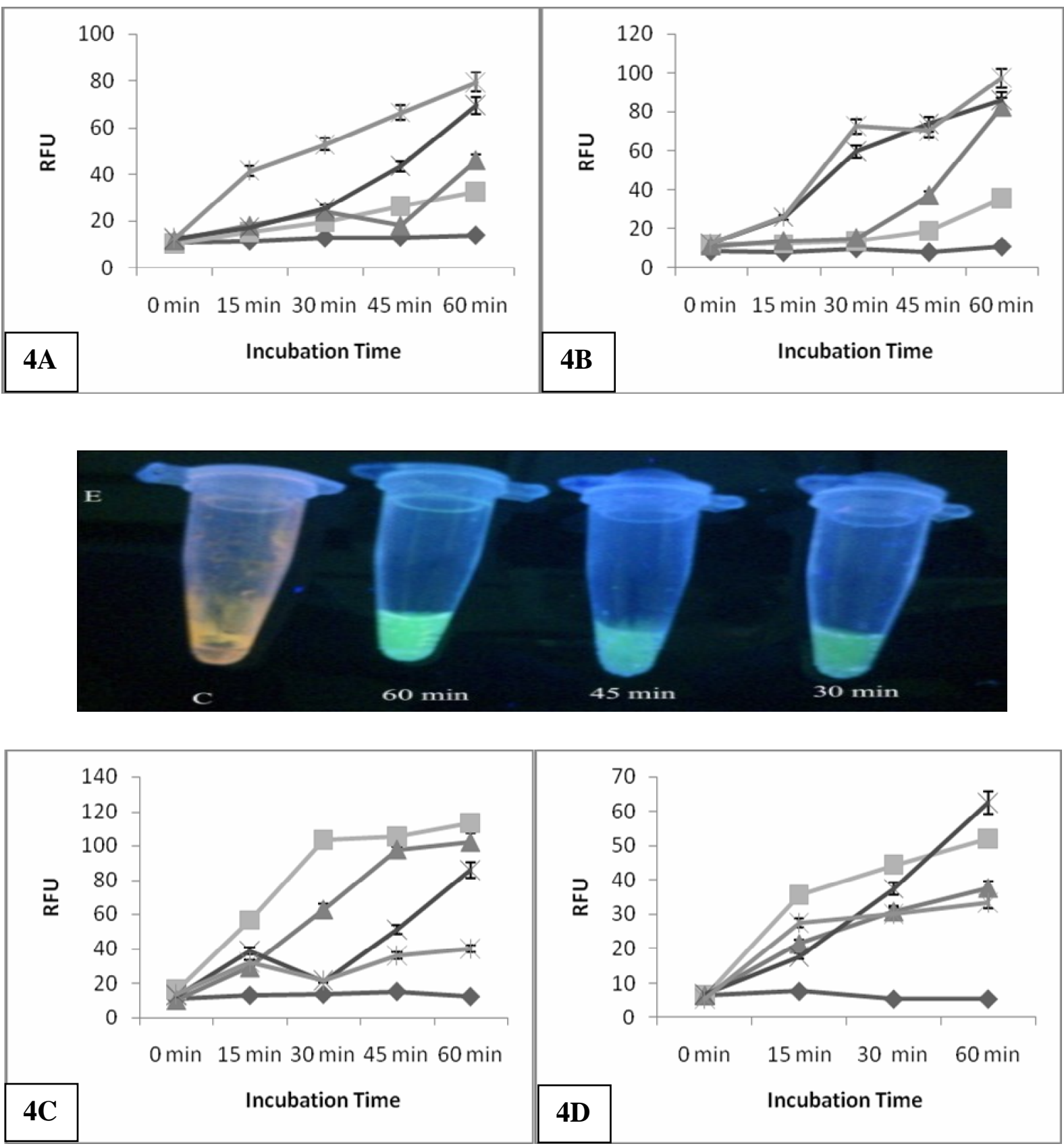

LAMP analysis of 4A) $h b l A$ gene; 4B) $b c e T$ gene; 4C) entFM/ cwpFM and 4D) $c y t K$ genes at 15 min intervals with varying concentrations of standard Bacillus cereus DNA.

$4 \mathrm{E})$ color change in tubes containing $10^{4}$ copies of DNA under UV light at varying incubation times: Tube C: control, Tube 1: $60 \mathrm{~min}$, Tube 2: $45 \mathrm{~min}$, Tube 3: $30 \mathrm{~min}$.

$(\diamond)$ Control, (ロ) $10^{1}$ copies, ( $\left.\mathbf{\Delta}\right) 10^{4}$ copies, (x) $10^{7}$ copies, (*) $10^{8}$ copies

Calcein: The colour change from orange (control) to bright yellow (amplified product) was in agreement with the agarose gel results (Fig.1). The colour change was difficult to be differentiated visually (Fig. $3 \mathrm{E})$. However, it could be read using a 
Varioskan Flash reader (absorption/emission spectra 495/517) and DNA concentrations as low as $10^{2}$ copies of DNA could be detected with Calcein dye (Fig. 3).

SYBR Green I: SYBR green I, a DNA intercalating dye, commonly used for LAMP analysis gave us good results and was sensitive upto $10^{2}$ copies of DNA (Fig. 4). The colour change from orange to green could be visually differentiated (Fig. 4E) and also could be quantified at $497 / 520 \mathrm{~nm}$ (absorption/emission spectra). On triplicate analysis, the instances of false positive results were observed thus hampered the reproducibility of the assay.

\section{Specificity of the LAMP Assay}

In order to evaluate and verify the specificity of the primers used in this study, each primer set was tested on DNA templates from 7 different bacterial test cultures (Table 1). The analysis indicated that all primers were specific for $B$. cereus diarrhealtoxin genes and there was no amplification due to cross reactivity with other target organisms (data not shown).

\section{Matrix Interference in Spiked Food Samples}

The effect of food matrix on the detection efficiency of LAMP assay was carried out with three dyes. The method developed involved spiking of food samples with reference B.cereus (MTCC 1272). The DNA was isolated by boiling in presence of triton X-100 (1.2\%) which resulted in good recovery of DNA from food samples. HNB dye showed positive colour change from violet to blue with all food matrices tested except milk and beetroot samples (Table 2, Sample No. 2 and 17). However, the same food matrices did not interfere when Calcein was used. Even though colour change in
Calcein from orange to yellow was not easy to differentiate with naked eyes, there was a marked increase in the absorption/emission spectra (495/ 517) of the spiked food samples when read using a Varioskan flash microplate reader. The colour change produced by SYBR Green I from orange to green (497/520) was equivocal as it produced false positive results in some control food matrices as well. Starchy food samples (Table 2, sample No: 8, 9, 16 and 21) showed positive amplification in case of all four toxigenic genes studied with negligible matrix interference. However, a few food samples such as raw beetroot, orange juice, panipuri, tomato and tomato sauce (Table 2, sample No. 17, 22, 18 and 25 ) interfered with the LAMP assay.

LAMP assay is highly specific as LAMP primers bind to six different regions on the target DNA. The assay can be carried out with both denatured and non-denatured DNA (Nagamine et al., 2001). However, denatured DNA template gave better amplification.

SYBR Green I fluoresces when it binds to the major grove of DNA. The SYBR Green dye, more often than not, binds to the primers and the template DNA present in the reaction mixture resulting in false positive results (Papin et al., 2010).

HNB and Calcein being metal chelator dyes detect the $\mathrm{Mg}^{2+}$ ion generated on amplification (Goto et al., 2009; Notomi et al., 2000b). In our studies we observed that the accuracy of HNB was dependent mainly on the presence of $\mathrm{Mg}^{2+}$ and the $\mathrm{pH}$ of the reaction mixture. The $\mathrm{pH}$ between $8.6-8.9$ was found to be optimum. HNB was found to be more acceptable for visual detection. The colour change was easily distinguishable with naked eyes. The food matrices that imparted colour or have an 
acidic $\mathrm{pH}$, however, interfered with the reaction sensitivity. This was especially true with beetroot and milk samples used in our study.

Calcein resulted in sensitive quantitative detection upon reading the fluorescence intensity in a Varioskan flash microplate reader. The colour change from orange to yellow although difficult to detect visually (Wastling et al., 2010) could be easily quantified in the microplate reader due to increase in the fluorescence as a result of the $\mathrm{Mg}^{2+}$ formation, a by-product of amplification reaction. Visual detection was however possible when a UV lamp was used to visualize the colour change.

LAMP reactions are generally performed using DNA isolates and have seldom been validated on direct spiked food samples. To validate the performance of LAMP reaction obtained using standard DNA, analysis was carried out with spiked food samples.

Contrary to the findings of Wastling et al., 2010, Calcein was found to be less prone to food matrix interference. Calcein equilibrated with $\mathrm{MnCl}_{2}$ only fluoresces when its $\mathrm{Mn}^{2+}$ ions are replaced with $\mathrm{Mg}^{2+}$ ions produced during amplification (Tomita et al., 2008b). Since the colour imparted by food matrix does not interfere with fluorescence of Calcein, it produced consistent results on analysis of spiked food samples. SYBR Green I showed false positive results with non- B. cereus spiked food sample as well. The dye intercalates with the DNA obtained from direct boiling method and primer dimers present in the reaction mixture and therefore produced a non-specific colour change. Also, Since SYBR Green I dye is added post reaction, the chances on contaminating the product is high (Chen et al., 2011), which is not the case with HNB and Calcein.
In conclusion, we observed $\mathrm{HNB}$ to be suitable for visual detection of $B$. cereus spiked food samples barring some food matrices that imparted colour. Calcein proved to be a better dye for detection of spiked food samples as the food matrix did not interfere with the detection sensitivity. The presence of $\mathrm{Hbl}$ toxin gene could be detected in all the food isolates tested, probably this is due to the predominance of $\mathrm{Hbl}$ toxin compared to other toxin genes as seen in our previous studies on the immunodetection of $B$. cereus $\mathrm{Hbl}$ toxin in food matrices (Machaiah and Krishnan 2014). To our knowledge, this is one of the first reports on LAMP based detection of Bacillus cereus. Work on development of an onsite detection kit for detection of Bacillus cereus in food and environmental samples are underway. Such a detection system will ensure better food safety and can also be extended to clinical diagnosis of pathogenic organisms.

\section{Acknowledgement}

The authors thank the Director, CSIRCentral Food Technological Research Institute for providing facilities to carry out the research work. The first author is grateful to the Indian Council of Medical Research (ICMR) for providing senior research fellowship to carry out the present work.

\section{References}

Burgess G, Horwood P (2006) Improved Molecular Detection Methods for Bacillus cereus Toxins.

Chen R, Tong Q, Zhang Y, Lou D, Kong Q, Lv S, Zhuo M, Wen L, Lu S (2011) Loop-mediated isothermal amplification: rapid detection of Angiostrongylus cantonensis 
infection in Pomacea canaliculata. Parasit Vectors 4:204. doi: 10.1186/1756-3305-4-204

Dierick K, Van Coillie E, Swiecicka I, Meyfroidt G, Devlieger H, Meulemans A, Hoedemaekers G, Fourie L, Heyndrickx M, Mahillon J (2005) Fatal family outbreak of Bacillus cereus-associated food poisoning. J Clin Microbiol 43:4277-9.

Ehling-schulz M, Fricker M, Scherer S (2004) Review Bacillus cereus, the causative agent of an emetic type of food-borne illness. 479-487. doi: 10.1002/mnfr.200400055

Goto M, Honda E, Ogura A, Nomoto A, Hanaki K-I (2009) Colorimetric detection of loop-mediated isothermal amplification reaction by using hydroxy naphthol blue. Biotechniques 46:167-72. doi: 10.2144/000113072

Gracias KS, McKillip JL (2011) Triplex PCR-based detection of enterotoxigenic Bacillus cereus ATCC 14579 in nonfat dry milk. J Basic Microbiol 51:147-52.

Granum PE (2005) Bacillus cereus. Foodborne Pathog. Microbiol. Mol. Biol.

Machaiah MI, Krishnan MH (2014) Immunodetection of Bacillus cereus haemolytic enterotoxin (HBL) in food samples. Anal Methods 6:1841. doi: 10.1039/c3ay41737a

Nagamine K, Watanabe K, Ohtsuka K, Hase T, Notomi T (2001) Loop-mediated Isothermal Amplification Reaction Using a Nondenatured Template. Clin Chem 47:1742-1743.

Notomi T, Okayama H, Masubuchi H, Yonekawa $\mathrm{T}$, Watanabe $\mathrm{K}$, Amino $\mathrm{N}$, Hase T (2000a) Loop-mediated isothermal amplification of DNA. Nucleic Acids Res 28:E63.
Notomi T, Okayama H, Masubuchi $\mathrm{H}$, Yonekawa T, Watanabe K, Amino $\mathrm{N}$, Hase T (2000b) Loop-mediated isothermal amplification of DNA. Nucleic Acids Res 28:E63.

Papin JF, Vahrson W, Larson L, Dittmer DP (2010) Genome-wide real-time PCR for West Nile virus reduces the falsenegative rate and facilitates new strain discovery. J Virol Methods 169:103-111.

Parida MM, Santhosh SR, Dash PK, Tripathi NK, Saxena P, Ambuj S, Sahni AK, Lakshmana Rao P V, Morita K (2006) Development and evaluation of reverse transcriptionloop-mediated isothermal amplification assay for rapid and real-time detection of Japanese encephalitis virus. J Clin Microbiol 44:4172-8.

Rajkovic A， Kljajic M, Smigic N, Devlieghere F, Uyttendaele M (2013) Toxin producing Bacillus cereus persist in ready-to-reheat spaghetti Bolognese mainly in vegetative state. Int J Food Microbiol 167:236-243.

Samapundo S, Heyndrickx M, Xhaferi R, Devlieghere F (2011) Incidence, diversity and toxin gene characteristics of Bacillus cereus group strains isolated from food products marketed in Belgium. Int $\mathbf{J}$ Food Microbiol 150:34-41. doi: 10.1016/j.ijfoodmicro.2011.07.013

Sambrook J, Fritsch EF, Maniatis T (1989) Molecular Cloning: A Laboratory Manual. Cold Spring Harbor laboratory press. New York 931957.

Schrader C, Schielke A, Ellerbroek L, Johne R (2012) PCR inhibitors-occurrence, properties and removal. J Appl Microbiol 113:1014-1026.

Service PH (2011) FDA Office of Foods- 
SRSC Method Vaildation Criteria.

Sowmya N, Thakur MS, Manonmani HK (2012) Rapid and simple DNA extraction method for the detection of enterotoxigenic Staphylococcus aureus directly from food samples: comparison of PCR and LAMP methods. J Appl Microbiol 113:10613.

Tomita N, Mori Y, Kanda H, Notomi T (2008a) Loop-mediated isothermal amplification (LAMP) of gene sequences and simple visual detection of products. Nat Protoc 3:877-882.

Tomita N, Mori Y, Kanda H, Notomi T (2008b) Loop-mediated isothermal amplification (LAMP) of gene sequences and simple visual detection of products. Nat Protoc 3:877-882.

Wang DG (2013) Rapid Detection of Mycobacterium tuberculosis Complex by Loop-Mediated Isothermal Amplification Combined with Chemosensor. Adv Mater Res 749:449-452.

Wastling SL, Picozzi K, Kakembo ASL, Welburn SC (2010) LAMP for human African trypanosomiasis: a comparative study of detection formats. PLoS Negl Trop Dis 4:e865.

\section{How to cite this article:}

Iychettira Machaiah Mandappa, Prasanna Joglekar, Munna Singh Thakur and Haravey Krishnan Manonmani. 2016. Loop-Mediated Isothermal Amplification (LAMP) Assay for Rapid Detection of Bacillus cereus Toxin Genes. Int.J.Curr.Microbiol.App.Sci. 5(1): 430-442 http://dx.doi.org/ $10.205 \overline{4} 6 /$ ijcmas. 2016.501 .043 